Pacific Journal of Mathematics

STRONG CONCENTRATION OF THE SPECTRA OF 


\title{
STRONG CONCENTRATION OF THE SPECTRA OF SELF-ADJOINT OPERATORS
}

\author{
CHRIS RORRES
}

\begin{abstract}
Let $H$ be a self-adjoint operator with spectral measure $E(S)$ over the Borel sets $S$ of the real line. The spectrum of $H$ is said to be strongly concentrated on $S$ if whenever $H_{n}$ converges strongly to $H$ in the generalized sense it is true that $E_{n}(S)$ converges strongly to the identity. Sufficient conditions on $H$ are given for this to occur for a given arbitrary Borel set $S$ and necessary and sufficient conditions when $S$ is the spectrum of $H$. In addition several more workable sufficient conditions are cited and a few examples illustrating the results are given.
\end{abstract}

Many authors have studied the changes in the spectra of a sequence of self-adjoint operators $H_{n}$ as it converges strongly in some sense to a self-adjoint operator-e.g., [2], [3], [5], [6], [7, pp. 471477], [8], [11]. It is known that while as point sets the spectra of $H_{n}$ do not necessarily converge to the spectrum of $H$, nevertheless in some sense the spectra of $H_{n}$ are concentrated on that of $H$. This spectral concentration phenomenon is described through the spectral measures $E_{n}, E$ of the operators involved. In particular since $E(\Sigma)$ is the identity when $\Sigma$ is the spectrum of $H$ it is reasonable to say that the spectrum of the sequence $H_{n}$ is concentrated on $\Sigma$ if $E_{n}(\Sigma)$ converges to the identity as $n \rightarrow \infty$. Our main results concern necessary and sufficient conditions for this to occur for an arbitrary sequence $H_{n}$ converging strongly to a fixed operator $H$. We make extensive use of the properties of the spectral measure $E(S)$ over the Borel sets $S$ of the real line for which a general reference is [4] $\S \S \mathrm{X} .2$ and XII. 2.

1. Preliminaries. Throughout this paper the following notation will be adhered to. $H$ will denote a self-adjoint operator over a Hilbert space $\boldsymbol{H}$. Its domain will be denoted by $D(H)$ and its spectrum by $\Sigma$ (which is always a closed subset of the real line $R$ ). The resolution of the identity of $H$ will be denoted by $E(\lambda),-\infty<\lambda<\infty$, and the associated projection-valued spectral measure by $E(S)$ over all Borel subsets $S$ of $R$. By convention we take $E(\lambda)$ to be right continuous, i.e., $E(\lambda+0)=E(\lambda)$. For a sequence of self-adjoint operators $H_{n}, n=1,2, \cdots$, over $\boldsymbol{H}$ the quantities $D\left(H_{n}\right), \Sigma_{n}, E_{n}(\lambda)$, and $E_{n}(S)$ are defined accordingly.

According to a definition of Rellich (cf. [9] or [7, p. 429]) we 
say that the sequence of self-adjoint operators $H_{n}$ coverges strongly to $H$ in the generalized sense (denoted $H_{n} \rightarrow H$ in the generalized sense) if there exists a dense linear manifold $D$ in $\boldsymbol{H}$ such that the following conditions are satisfied:

(i) $D \subseteq D\left(H_{n}\right)$ for all $n$ sufficiently large

(ii) the closure of $H$ restricted to $D$ is again $H$

(iii) $\lim _{n \rightarrow \infty} H_{n} u=H u$ for all $u \in D$.

If the operators $H_{n}$ and $H$ are all bounded the above definition reduces to ordinary strong convergence which we denote simply $H_{n} \underset{s}{\rightarrow} H$.

The following theorem of Rellich (cf. [9] or [7, p. 432]) will be basic to our analysis:

THEOREM 1.1. Let the sequence of self adjoint operators $H_{n}$ converge strongly in the generalized sense to the self-adjoint operator $H$. Then if $\lambda$ is not an eigenvalue of $H$ we have

$$
E_{n}(\lambda) \underset{s}{\longrightarrow} E(\lambda)
$$

and

$$
E_{n}(\lambda-0) \underset{s}{\longrightarrow} E(\lambda)
$$

Next we give the definition of a spectral concentration phenomenon suggested by Titchmarsh (cf. [11], [12, p. 261]) and later refined by Conley and Rejto (cf. [2], [3]).

DEFINITION $1.2^{1}$. The spectrum of $H_{n}$ is asymptotically concentrated on the Borel set $S$ if $E_{n}(S) \underset{s}{\rightarrow} I$.

Asymptotic concentration is thus a property of a sequence $H_{n}$ (and a subset $S$ ). We now introduce the definition of a concentration phenomenon associated with a single self-adjoint operator $H$.

DEFINITION 1.3. The spectrum of $H$ is strongly concentrated on the Borel set $S$ if whenever $H_{n} \underset{s}{\rightarrow} H$ in the generalized sense it is true that $E_{n}(S) \rightarrow I$.

Hence if the spectrum of $H$ is strongly concentrated on $S$ then the spectrum of any sequence $H_{n}$ which converges strongly to $H$ in the generalized sense is asympotically concentrated on $S$.

The following lemma states, as we would expect, that if the

1 Actually asymptotic concentration is defined more generally to allow the subset $S$ to depend on $n$. We then say the spectrum of $H_{n}$ is asymptotically concentrated on

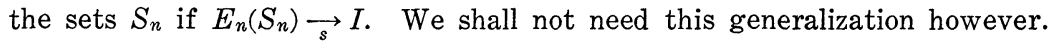


spectrum of an operator is strongly concentrated on a set it is also strongly concentrated on any larger set. The proof follows easily from the fact that if $S \subseteq S^{\prime}$ then $E(S) \leqq E\left(S^{\prime}\right)$.

LEMMA 1.4. If the spectrum of $H$ is strongly concentrated on $S$ and if $S \subseteq S^{\prime}$ then the spectrum of $H$ is strongly concentrated on $S^{\prime}$.

2. Main results. Our main interest will be to see how small we may make the set $S$. To this end the following theorem (cf. [7, p. 472]), reworded using our terminology, is of interest:

THEOREM 2.1. Let $S$ be an open set which contains the spertrum $\Sigma$ of $H$. Then the spectrum of $H$ is strongly concentrated on $S$.

We shall next strengthen this theorem to the case where $S$ is not necessarily open. Let int $S$ denote the interior of the set $S$ and let $\partial S$ denote its boundary. Then

THEOREM 2.2. Let $S$ be a Borel set which contains the spectrum $\Sigma$ of $H$. Then if $E(\partial S)=0$ the spectrum of $H$ is strongly concentrated on int $S$.

Proof. We have

$$
\Sigma \subseteq S \subseteq(\operatorname{int} S) \cup \partial S \text { and (int } S) \cap \partial S=\varnothing
$$

hence

$$
E(\Sigma) \leqq E(S) \leqq E(\text { int } S)+E(\partial S) \cdot
$$

But $E(\Sigma)=I$ and $E(\partial S)=0$. Hence $E($ int $S)=I$.

Since int $S$ is an open subset of $R$ it may be expressed as a countable union of disjoint open intervals, say

$$
\operatorname{int} S=\bigcup_{k=1}^{\infty} I_{k} \text { where } I_{k}=\left(\alpha_{k}, \beta_{k}\right) .
$$

Since the spectral measure is strongly countably additive we therefore have

$$
\sum_{k=1}^{\infty} E\left(I_{k}\right)=I .
$$

Furthermore none of the endpoints of the intervals $\left(\alpha_{k}, \beta_{k}\right)$ can be eigenvalues of $H$, for the endpoints belong to $\partial S$ and $E(\partial S)=0$ while for eigenvalues $\lambda_{0}$ we have $E\left(\left\{\lambda_{0}\right\}\right) \neq 0$. Since (1) is in the sense of strong convergence of the sum, given any $u \in \boldsymbol{H}$ and any $\varepsilon>0$ 
there exists a $K$ such that

$$
\left\|\left(I-\sum_{k=1}^{K} E\left(I_{k}\right)\right) u\right\|<\varepsilon / 2 .
$$

Now let $H_{n}$ be any sequence which converges strongly to $H$ in the generalized sense. Then by Theorem 1.1 we have

$$
E_{n}\left(\beta_{k}-0\right) \underset{s}{\longrightarrow} E\left(\beta_{k}\right) \quad \text { as } n \longrightarrow \infty
$$

and

$$
E_{n}\left(\alpha_{k}\right) \underset{s}{\longrightarrow} E\left(\alpha_{k}\right) \quad \text { as } n \longrightarrow \infty \text {. }
$$

For the above value of $K$ we may therefore find a value $N$ such that for $n \geqq N$

$$
\begin{aligned}
& \left\|\left(E_{n}\left(\beta_{k}-0\right)-E\left(\beta_{k}\right)\right) u\right\|<\varepsilon / 4 K \\
& \left\|\left(E_{n}\left(\beta_{k}-0\right)-E\left(\alpha_{k}\right)\right) u\right\|<\varepsilon / 4 K
\end{aligned}
$$

for $k=1,2, \cdots, K$.

By definition of the spectral measure we have

$$
E\left(I_{k}\right)=E\left(\beta_{k}\right)-E\left(\alpha_{k}\right) \quad\left(\text { since } E\left(\beta_{k}-0\right)=E\left(\beta_{k}\right)\right)
$$

and

$$
E_{n}\left(I_{k}\right)=E_{n}\left(\beta_{k}-0\right)-E_{n}\left(\alpha_{k}\right) \text {. }
$$

Hence by successive use of the triangle inequality we have for $n \geqq N$

$$
\begin{aligned}
& \left\|\left(I-\sum_{k=1}^{K} E_{n}\left(I_{k}\right)\right) u\right\| \leqq\left\|\left(I-\sum_{k=1}^{K} E\left(I_{k}\right)\right) u\right\| \\
& \quad+\left\|\sum_{k=1}^{K} E\left(I_{k}\right) u-\sum_{k=1}^{K} E_{n}\left(I_{k}\right) u\right\|<\varepsilon / 2+\sum_{k=1}^{K}\left\|\left(E\left(\beta_{k}\right)-E_{n}\left(\beta_{k}-0\right)\right) u\right\| \\
& \quad+\sum_{k=1}^{K}\left\|\left(E_{n}\left(\alpha_{k}\right)-E\left(\alpha_{k}\right)\right) u\right\|<\varepsilon / 2+\sum_{k=1}^{K} \varepsilon / 4 K+\sum_{k=1}^{K} \varepsilon / 4 K=\varepsilon .
\end{aligned}
$$

Now for all $K$ we have

$$
\bigcup_{k=1}^{K} I_{k} \subseteq \bigcup_{k=1}^{\infty} I_{k}=\operatorname{int} S
$$

hence

$$
\sum_{k=1}^{K} E_{n}\left(I_{k}\right) \leqq E_{n}(\operatorname{int} S)
$$

and so 


$$
I-E_{n}(\operatorname{int} S) \leqq I-\sum_{k=1}^{K} E_{n}\left(I_{k}\right)
$$

Therefore from (2) above we have for $n \geqq N$

$$
\left\|\left(I-E_{n}(\operatorname{int} S)\right) u\right\|<\varepsilon
$$

which implies that $E_{n}($ int $S) \underset{s}{\rightarrow} I$.

Notice that Theorem 2.2 is indeed a generalization of Theorem 2.1 for if $S$ is open then int $S=S$ and if $\Sigma \subseteq S$ then $\partial S$ must lie in the resolvent set of $H$, hence $E(\partial S)=0$.

This theorem suggests that under certain conditions we may expect the spectrum of $H$ to be strongly concentrated on itself, by which we mean

Definition 2.3. The spectrum $\Sigma$ of $H$ is strongly concentrated on itself if whenever $H_{n} \underset{s}{\rightarrow} H$ in the generalized sense it is true that $E_{n}(\Sigma) \rightarrow I$.

The condition suggested by Theorem 2.2 will be shown also to be necessary in the following

THEOREM 2.4. The spectrum $\Sigma$ of $H$ is strongly concentrated on itself if and only if $E(\partial \Sigma)=0$.

Proof. First assume that $E(\partial \Sigma)=0$. Then from Theorem 2.2 with $S=\Sigma$ we have that the spectrum of $H$ is strongly concentrated on int $\Sigma$ hence on itself.

To prove the converse we must show that if $E(\partial \Sigma) \neq 0$ then there exists a sequence $H_{n}$ which converges strongly to $H$ in the generalized sense for which $E_{n}(\Sigma)$ does not converge strongly to the identity. To construct this sequence we will need the following subspaces

$$
\boldsymbol{H}_{B}=E(\partial \Sigma) \boldsymbol{H} \text { and } \boldsymbol{H}_{I}=E(\operatorname{int} \Sigma) \boldsymbol{H} .
$$

Since $\Sigma=\operatorname{int} \Sigma \cup \partial \Sigma$ and int $\Sigma \cap \partial \Sigma=\varnothing$ the closed subspaces $\boldsymbol{H}_{B}$ and $\boldsymbol{H}_{I}$ are orthogonal and span the whole space $\boldsymbol{H}$. Furthermore they each reduce the operator $H$. Let us denote the part of $H$ restricted to $\boldsymbol{H}_{B}$ by $H_{B}$ and the part of $H$ restricted to $\boldsymbol{H}_{I}$ by $H_{I}$. Let $\Sigma_{B}$ and $\Sigma_{I}$ be their corresponding spectra. Then we have $\Sigma=\Sigma_{B} \cup \Sigma_{I}$, $\Sigma_{B} \subseteq \partial \Sigma$, and $\Sigma_{I}=\overline{\operatorname{int} \Sigma}$ where $\overline{\operatorname{int} \Sigma}$ is the closure of the set int $\Sigma$.

We may now define the sequence $H_{n}$ in each of the subspaces $\boldsymbol{H}_{B}$ and $\boldsymbol{H}_{I}$. We set 


$$
H_{I . n} u=H u \text { for } u \in \boldsymbol{H}_{I} \cap D(H)
$$

and

$$
H_{B, n} u=\sum_{k=-\infty}^{\infty} \lambda_{k n} E\left(I_{k n}\right) u \quad \text { for } u \in \boldsymbol{H}_{B} \cap D(H) \text {. }
$$

$H_{B, n}$ is an approximation to the representation $H=\int \lambda d E(\lambda)$ restricted to $\boldsymbol{H}_{B}$, which converges strongly to $H_{B}$ in the generalized sense as $n \rightarrow \infty$. For each fixed $n$ the intervals $I_{k n}, k=0, \pm 1, \cdots$, are to be a subdivision of the real line chosen so that the end points do not fall on $\partial \Sigma$. The length of the largest interval is to approach zero as $n \rightarrow \infty$. Each interval $I_{k n}$ which contains a point of $\partial \Sigma$ also contains points not belonging to $\Sigma$, from which we choose $\lambda_{k n}$. In the intervals which do not contain points of $\partial \Sigma$ we have that $E\left(I_{k n}\right) u=$ 0 for $u \in \boldsymbol{H}_{B} \cap D(H)$, hence the choice of $\lambda_{k n}$ is immaterial. The spectrum of $H_{B, n}$ for each fixed $n$ consists of those $\lambda_{k n}, k=0, \pm 1, \cdots$, for which $\partial \Sigma \cap I_{k n} \neq \varnothing$, and so is disjoint from $\Sigma$.

Finally let

$$
H_{n}=H_{I, n} \oplus H_{B, n} \text {. }
$$

Then $H_{n s} \rightarrow H$ in the generalized sense since $H_{I, n} u \rightarrow H_{I} u$ and $H_{B, n} v \rightarrow$ $H_{B} v$ for all $u \in \boldsymbol{H}_{I} \cap D(H)$ and all $v \in H_{B} \cap D(H)$. To show that $E_{n}(\Sigma)$ does not converge strongly to the identity, let $v$ be any nonzero element of $\boldsymbol{H}_{B}$. Then

$$
E_{n}(\Sigma) v=E_{B, n}(\Sigma) v \text {. }
$$

But the spectrum of $H_{B, n}$ is disjoint from $\Sigma$, hence $E_{B, n}(\Sigma)=0$. Therefore $E_{n}(\Sigma) v=0$ and so $E_{n}(\Sigma) v$ does not converge to $v$.

Actually this theorem also shows the spectrum of $H$ to be strongly concentrated on a slightly smaller set, as follows.

CoRollary 2.5. The spectrum $\Sigma$ of $H$ is concentrated on itself if and only if it is concentrated on int $\Sigma$.

Proof. The "if" part follows from Lemma 1.4. Conversely if the spectrum of $H$ is concentrated on itself then $\mathrm{E}(\partial \Sigma)=0$ and hence from Theorem 2.2 with $S=\Sigma$ it is concentrated on int $\Sigma$.

The condition $E(\partial \Sigma)=0$ requires that no eigenvalues of $H$ lie on $\partial \Sigma$ (in particular $H$ may have no isolated eigenvalues). However more is required. To give sufficient conditions for $E(\partial \Sigma)=0$ let us denote by $\boldsymbol{H}_{A C}$ the set of all $u \in \boldsymbol{H}$ for which $(E(\lambda) u, u)$ is absolutely 
continuous in $\lambda$. It is known (cf. $\left[7\right.$, p. 516]) that $\boldsymbol{H}_{A C}$ is a closed subspace of $\boldsymbol{H}$ which reduces $H$. Let us further denote by $m(S)$ the Lebesgue measure of the set $S$. Then we have

THEOREM 2.6. If $m(\partial \Sigma)=0$ and $\boldsymbol{H}_{B} \subseteq \boldsymbol{H}_{A C}$ then $E(\partial \Sigma)=0$.

Proof. If $u \in \boldsymbol{H}_{I}$ then $E($ int $\Sigma) u=u$. Hence

$$
E(\partial \Sigma) u=E(\partial \Sigma) E(\operatorname{int} \Sigma) u=0 \text { since } \partial \Sigma \cap \text { int } \Sigma=\varnothing \text {. }
$$

And if $u \in \boldsymbol{H}_{B}$ then $u \in \boldsymbol{H}_{A C}$ and since then $(E(\lambda) u, u)$ is absolutely continuous we have

$$
\|E(\partial \Sigma) u\|^{2}=(E(\partial \Sigma) u, u)=\int_{\partial \Sigma} d(E(\lambda) u, u)=0
$$

as $m(\partial \Sigma)=0$.

As $\boldsymbol{H}_{I}$ and $\boldsymbol{H}_{B}$ span $\boldsymbol{H}$ we have $E(\partial \Sigma) u=0$ for all $u \in \boldsymbol{H}$.

The orthogonal complement of $\boldsymbol{H}_{A C}$ is denoted by $\boldsymbol{H}_{S}$ and is identical to the set of all $u \in \boldsymbol{H}$ for which $(E(\lambda) u, u)$ is a singular function of $\lambda$. The spectrum of $H$ restricted to $\boldsymbol{H}_{A C}$ or $\boldsymbol{H}_{S}$ is called, respectively, the absolutely continuous spectrum and the singular spectrum of $H$ (denoted $\Sigma_{A C}$ and $\Sigma_{S}$ ). The condition $\boldsymbol{H}_{B} \subseteq \boldsymbol{H}_{A C}$ in Theorem 2.6 implies, but is not implied by, the condition $\partial \Sigma \cong \Sigma_{A C}$. The following corollary gives sufficient conditions for $E(\partial \Sigma)=0$ in terms of the singular spectrum.

CoRollary 2.7. If $m(\partial \Sigma)=0$ and $\partial \Sigma \cap \Sigma_{S}=\varnothing$ then $E(\partial \Sigma)=0$.

Proof. If $\partial \Sigma \cap \Sigma_{S}=\varnothing$ then $E(\partial \Sigma) E\left(\Sigma_{S}\right)=0$ and hence $E(\partial \Sigma) \boldsymbol{H}$ and $E\left(\Sigma_{S}\right) \boldsymbol{H}$ are orthogonal subspaces. Now $\boldsymbol{H}_{B}=E(\partial \Sigma) \boldsymbol{H}$ and $\boldsymbol{H}_{S} \subseteq$ $E\left(\Sigma_{S}\right) \boldsymbol{H}$ since $\boldsymbol{H}_{S}$ reduces $H$, consequently $\boldsymbol{H}_{B}$ and $\boldsymbol{H}_{S}$ are orthogonal. But since $\boldsymbol{H}_{A C}$ is the orthogonal complement of $\boldsymbol{H}_{S}$ it follows that $\boldsymbol{H}_{B} \subseteq \boldsymbol{H}_{A C}$ and so the conditions of Theorem 2.6 are satisfied.

A weaker but somewhat more useful sufficient condition is the following:

THEOREM 2.8. Let $\partial \Sigma$ be countable and not contain any eigenvalues of $H$. Then $E(\partial \Sigma)=0$.

Proof. Let $\partial \Sigma=\mathbf{U}_{k=1}^{\infty}\left\{\lambda_{k}\right\}$. Then

$$
E(\partial \Sigma)=\sum_{k=1}^{\infty} E\left(\left\{\lambda_{k}\right\}\right)=\sum_{k=1}^{\infty}\left(E\left(\lambda_{k}\right)-E\left(\lambda_{k}-0\right)\right)=0
$$


since $E(\lambda)$ is continuous at any point $\lambda$ not an eigenvalue of $H$.

3. Examples and applications. Our first example will be to show that the condition $m(\partial \Sigma)=0$ in theorem 2.6 is essential. Let $\left\{r_{n}\right\} n=1,2, \cdots$, be an enumeration of the rational numbers in the closed interval $[0,1]$. Let $\varepsilon$ be any positive number less than 1 and let

$$
0_{n}=\left\{x \in(0,1)|| x-r_{n} \mid<\varepsilon / 2^{n}\right\}
$$

and

$$
Q=\bigcup_{n=1}^{\infty} 0_{n}
$$

Then $0_{n}$ is an open set with Lebesgue measure less than or equal to $\varepsilon / 2^{n}$, and $Q$ is open with

$$
m(Q) \leqq \sum_{n=1}^{\infty} m\left(0_{n}\right) \leqq \sum_{n=1}^{\infty} \varepsilon / 2^{n}=\varepsilon .
$$

If we set

$$
P=([0,1]-Q) \cup\{0\} \cup\{1\}
$$

then $P$ is a closed nowhere dense subset of the unit interval consisting entirely of irrational numbers (plus the endpoints 0 and 1). Furthermore $m(P) \geqq 1-\varepsilon$. We define a Borel measure $\sigma$ on $R$ by

$$
\sigma(S)=m(S \cap P)
$$

with associated generating function

$$
\sigma(x)=\sigma((-\infty, x)) .
$$

Our Hilbert space $\boldsymbol{H}$ will be $L_{\sigma}^{2}(R)$, consisting of all $\sigma$-measurable functions $f(x)$ on $R$ for which

$$
\int|f(x)|^{2} d \sigma(x)<\infty
$$

The multiplication operator

$$
(H f)(x)=x f(x)
$$

is then self-adjoint on $H$ with spectrum $\Sigma=P$ (cf. [1, pp. 103-106]). But $P$ is closed and nowhere dense, hence $\partial P=\partial \Sigma=\Sigma$. Hence $E(\partial \Sigma)=E(\Sigma)=I$ and so the spectrum of $H$ cannot be concentrated on itself. Furthermore since $\sigma$ is a restriction of Lebesgue measure it is absolutely continuous, hence $\boldsymbol{H}_{A C}=\boldsymbol{H}_{B}=\boldsymbol{H}$. 
Our second example will be to show that the condition $\boldsymbol{H}_{B} \subseteq \boldsymbol{H}_{A C}$ is also essential in Theorem 2.6. Let $c(x)$ be the Cantor Ternary function (cf. [10, p. 39]) on the unit interval and let $c(S)$ be the associated Cantor measure on $R$. Our Hilbert space will be $L_{c}^{2}(R)$ and $H$ will again be multiplication by the independent variable. $c(x)$ is a continuous non-absolutely continuous function whose only points of increase are on the Cantor set $C$. Hence $\Sigma=C$. But $C$ is closed and nowhere dense so that $\partial C=\partial \Sigma=\Sigma$. Therefore $E(\partial \Sigma)=E(\Sigma)=$ $I$ and so the spectrum of $H$ cannot be strongly concentrated on itself. And since the Cantor set has Lebesgue measure zero we have $m(\Sigma)=0$. The theorem fails, of course, since $H$ has no absolutely continuous spectrum and $\boldsymbol{H}_{B}=\boldsymbol{H}$.

Our final example will be a positive one of interest in itself. Let $\boldsymbol{H}=L^{2}(R)$ and let $H$ be the Schroedinger operator

$$
(H f)(x)=-\frac{d^{2} f}{d x^{2}}+g(x) f
$$

acting on the class of functions $f(x)$ with absolutely continuous first derivatives for which $H f \in \boldsymbol{H}$. Here $g(x)$ is a continuous real-valued periodic function. $H$ is the Hamiltonian operator of a one-dimensional quantum mechanical particle moving in a periodic potential (a crystal for example). It is known (cf. [12, Chapter XXI]) that $H$ is selfadjoint with a purely continuous spectrum consisting of a sequence of closed intervals bounded below, extending to $+\infty$, and separated by a finite or infinite number of gaps (these are the so-called energy bands of solid state physics). Since the conditions of Theorem 2.8 are satisfied the spectrum of $H$ is strongly concentrated on itself.

Let us consider the following sequence of operators:

$$
\left(H_{n} f\right)(x)=-\frac{d^{2} f}{d x^{2}}+g_{n}(x) f
$$

where

$$
g_{n}(x)=\left\{\begin{array}{rl}
g(x) & \text { for }|x| \leqq n \\
0 \text { for }|x|>n
\end{array} .\right.
$$

The operators $H_{n}$ are self-adjoint over the same domain as $H$ and they converge strongly to $H$ in the generalized sense since for $f \in D(H)=D\left(H_{n}\right)$ we have

$$
\left\|H f-H_{n} f\right\|^{2}=\int_{|x|>n}|g(x)|^{2}|f(x)|^{2} d x \longrightarrow 0 \text { as } n \longrightarrow \infty .
$$

The operator $H_{n}$ is the Hamiltonian operator of a particle moving in a crystal of finite extent. Its spectrum, since $g_{n}(x)$ is continuous 
with compact support, consists of a continuous portion $[0, \infty)$ and at most a finite number of negative eigenvalues with finite multiplicity.

The quantity $\|E(S) f\|^{2}$ in quantum mechanics represents the probability of measuring the value of the energy of the particle in the state $f$ within the subset $S$. While for a finite crystal the energy may assume any value from 0 to $+\infty$, for an infinite crystal the energy must lie within the energy bands of the operator $H$. The fact that the spectrum of $H$ is strongly concentrated on itself then assures us that for a finite crystal and a fixed state $f$ we may make the probability of finding the energy outside the energy bands of the infinite crystal as small as we desire by taking the crystal sufficiently large (i.e., by choosing $n$ sufficiently large).

\section{REFERENCES}

1. N. I. Akhiezer and I. M. Glazman, Theory of Linear Operators in Hilbert Space, Vol. I, (English translation). Ungar, New York, 1961.

2. C. C. Conley and P. A. Rejto, On spectral concentration, New York University, Courant Inst. Math. Sci., Res. Rep. No. IMM-NYU-293, 1962.

3. - Spectral concentration II-General Theory, Perturbation Theory and its Applications in Quantum Mechanics, ed. C. H. Wilcox, Wiley, New York, 1966.

4. N. Dunford and J. T. Schwartz, Linear Operators, Part II. Spectral Theory of Self adjoint Operators in Hilbert Space, Interscience, New York, 1963.

5. K. O. Friedrichs and P. A. Rejto, On a perturbation through which the discrete spectrum becomes continuous, Comm. Pure Appl. Math., 15 (1962), 219-235.

6. T. Kato, On the convergence of the perturbation method, J. Fac. Sci. Univ. Tokyo, 6 (1951), 145-226.

7. - Perturbation Theory for Linear Operators, Springer, New York, 1966.

8. J. B. McLeod, "Spectral concentration I-the one-dimensional Schroedinger operator", Perturbation Theory and its Applications in Quantum Mechanics, ed. C. H. Wilcox, Wiley, New York, 1966.

9. F. Rellich, Störungstheorie der Spektralzerlegung. II, Math. Ann., 113 (1936), 677-685.

10. H. L. Royden, Real Analysis, Macmillan, New York, 1963.

11. E. C. Titchmarsh, Some theorems on perturbations, V, J. Analyse Math., 4 (1954/55), 187-208.

12. - Eigenfunction Expansions Associated with Second Order Differential Equations, Part II, Clarendon Press, Oxford, 1958.

Received July 23, 1970.

Drexel University 


\section{PACIFIC JOURNAL OF MATHEMATICS}

\section{EDITORS}

H. SAMELSON

Stanford University

Stanford, California 94305

C. R. Hовву

University of Washington

Seattle, Washington 98105
J. DugundJI

Department of Mathematics

University of Southern California

Los Angeles, California 90007

RICHARD ARENS

University of California

Los Angeles, California 90024

\section{ASSOCIATE EDITORS}
E. F. BECKENBACH
B. H. NeumanN
F. WOLF
K. YOSHIDA

\section{SUPPORTING INSTITUTIONS}

UNIVERSITY OF BRITISH COLUMBIA

CALIFORNIA INSTITUTE OF TECHNOLOGY

UNIVERSITY OF CALIFORNIA

MONTANA STATE UNIVERSITY

UNIVERSITY OF NEVADA

NEW MEXICO STATE UNIVERSITY

OREGON STATE UNIVERSITY

UNIVERSITY OF OREGON

OSAKA UNIVERSITY
UNIVERSITY OF SOUTHERN CALIFORNIA

STANFORD UNIVERSITY

UNIVERSITY OF TOKYO

UNIVERSITY OF UTAH

WASHINGTON STATE UNIVERSITY

UNIVERSITY OF WASHINGTON

$\stackrel{*}{*} \stackrel{*}{*} \stackrel{*}{*}{ }^{*}$ AMERICAN MATHEMATICAL SOCIETY

NAVAL WEAPONS CENTER 


\section{Pacific Journal of Mathematics}

\section{Vol. 41, No. $1 \quad$ November, 1972}

Anatole Beck and Peter Warren, Weak orthogonality.................

Jonnie Bee Bednar and Howard E. Lacey, Concerning Banach spaces whose duals are abstract $L$-spaces.............................

Louis Harvey Blake, Canonical extensions of measures and the extension of regularity of conditional probabilities..........................

R. A. Brooks, Conditional expectations associated with stochastic processes ..........................................

Theodore Allen Burton and Ronald Calvin Grimmer, On the asymptotic behavior of solutions of $x^{\prime \prime}+a(t) f(x)=e(t) \ldots \ldots \ldots \ldots \ldots \ldots$

Stephen LaVern Campbell, Operator-valued inner functions analytic on the closed disc ............................................

Yuen-Kwok Chan, A constructive study of measure theory...

Alexander Munro Davie and Bernt Karsten Oksendal, Peak interpolation sets for some algebras of analytic functions ...................

H. P. Dikshit, Absolute total-effective $\left(N, p_{n}\right)(c, 1)$ method ...............

Robert E. Edwards, Edwin Hewitt and Kenneth Allen Ross, Lacunarity for

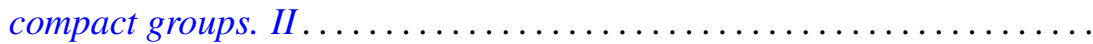

James Daniel Halpern, On a question of Tarski and a maximal theorem of Kurepa

Gerald L. Itzkowitz, A characterization of a class of uniform spaces that admit an invariant integral

Mo Tak Kiang, Semigroups with diminishing orbital diameters ..

Glenn Richard Luecke, A class of operators on Hilbert space ...

R. James Milgram, Group representations and the Adams spectral sequence. . .

G. S. Monk, On the endomorphism ring of an abelian p-group, and of a large subgroup...

Yasutoshi Nomura, Homology of a group extension ...

R. Michael Range, Approximation to bounded holomorphic functions on strictly pseudoconvex domains...

Norman R. Reilly, Inverse semigroups of partial transformations and $\theta$-classes.

Chris Rorres, Strong concentration of the spectra of self-adjoint operators

Saharon Shelah, A combinatorial problem; stability and order for models and theories in infinitary languages.

George Gustave Weill, Vector space decompositions and the abstract

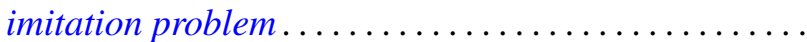

